\title{
Anti-slug control solutions based on identified model
}

\author{
Esmaeil Jahanshahi, Sigurd Skogestad \\ Department of Chemical Engineering, Norwegian University of Science and Technology (NTNU), NO-7491 \\ Trondheim (e-mail: skoge@ntnu.no)
}

\begin{abstract}
A anti-slug control requires operation around an open-loop unstable operating point. One solution is to design a robust controller based on a mechanistic model. An alternative and more robust approach is to identify an unstable model of the system based on input-output data. We used a closed-loop step test to identify an unstable linear model. From this, we obtained a second order IMC (Internal Model Control) controller that can be implemented as a PIDF controller. From the asymptotes of the proposed IMC controller, we also derive a simple tuning for PI-controller. Next, we considered two types of robust $\mathcal{H}_{\infty}$ controller (mixed-sensitivity and loop-shaping). The proposed model identification and control solutions were verified experimentally on two different test rigs. We found that the robustness and performance of the IMC (PIDF) controller is comparable with the $\mathcal{H}_{\infty}$ controllers. However, the prosed IMC (PIDF) controller is easier to tune compared to $\mathcal{H}_{\infty}$ control.
\end{abstract}

Keywords: Oil production, anti-slug control, unstable systems, robust control 2014 MSC: JPROCONT-D-14-00203R1

\section{Introduction}

Severe slugging flow regimes usually occur in pipeline-riser systems that transport oil and gas mixture from the seabed to the surface (Yocum, 1973). Such flow regimes, also referred to as "riser slugging", are characterised by severe flow and pressure oscillations.

Slugging has been recognised as a serious problem in offshore oilfields, because the irregular flow caused by slugging can cause serious operational problems for the downstream surface facilities (e.g. overflow of inlet separators). Therefore, effective ways to handle or remove riser slugging are needed, and many efforts have been made in order to prevent such occurrences (Courbot, 1996; Havre et al., 


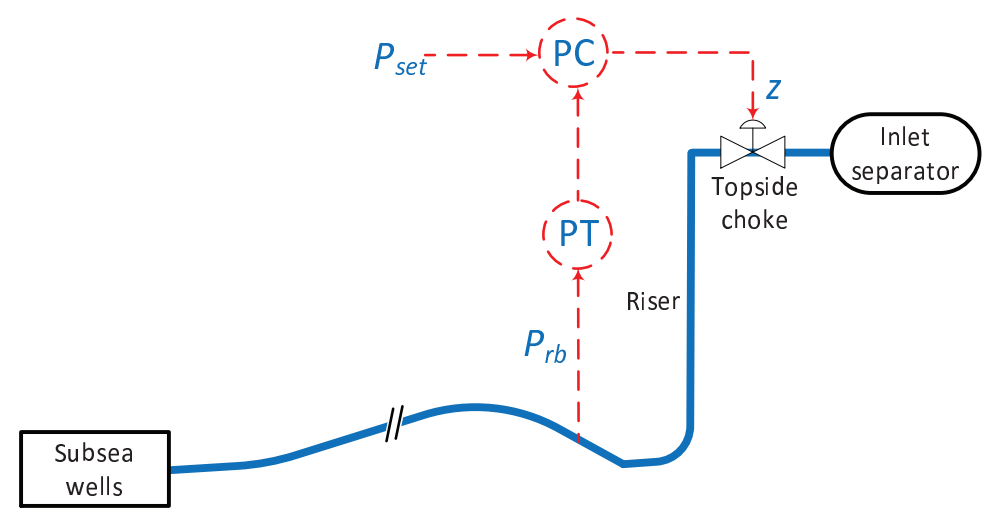

Figure 1: Preventing slug flow by control of riser base pressure $\left(M V=Z, C V=P_{r b}\right)$

2000). The conventional solution is to reduce the opening of the top-side choke valve (choking), but this may reduce the production rate especially for fields where the reservoir pressure is relatively low. Therefore, a solution that guarantees stable flow together with the maximum possible production rate is desirable.

Fortunately, automatic feedback control has been shown to be an effective strategy to eliminate the slugging problem (Havre et al., 2000; Godhavn et al., 2005). As shown in Figure 1, the top-side choke valve is usually used as the manipulated variable to regulate (control) the riser base pressure $\left(P_{\mathrm{rb}}\right)$ at a given pressure set-point $\left(P_{\mathrm{set}}\right)$. Such a system is referred to as 'anti-slug control' and it aims at stabilising the flow in the pipeline at operating conditions that, without control, would lead to riser slugging.

The system nonlinearity, inflow disturbances and plant changes/uncertianties make the slugging a challenging control problem. The nonlinearity of the system is one problem for a linear controller, because the gain of the system changes drastically at different operating conditions. In addition, the effective time delay is another problematic factor for stabilization. The main objective of our research is to design robust anti-slug control systems to prevent the slugging.

One solution is to use nonlinear model-based controllers to counteract the nonlinearity (e.g. Di Meglio et al. (2010)). However, we have found that these solutions are less robust against time delays or plant/model mismatch (Jahanshahi and Skogestad, 2013b).

An alternative approach is to identify an unstable model of the system, for example, using a closed-loop step test. We use the identified model for an IMC (Internal Model Control) design, 
which in our case can be realized as a PIDF controller. We define a PIDF controller as

$$
K_{P I D F}(s)=K_{c}\left(1+\frac{1}{s T_{i}}+\frac{T_{d} s}{T_{f} s+1}\right)
$$

where $K_{p}$ is the proportional gain, $T_{i}$ is the integral time, $T_{d}$ is the derivative time and $T_{f}$ is the time constant of the derivative action filter. We differentiate this from a PID controller (with a filter), because the low-pass filter is a crucial part of the controller for our application, not just to reduce the noise effect. That is we cannot set $T_{f}$ to a small value and obtain the same or better performance.

As the simpler alternative solution, we consider PI-control, which is the preferred choice in the industry. However, appropriate settings are required for robustness, and we obtain the PI-controller settings from the asymptotes of the proposed IMC controller.

Finally, we consider two different robust $\mathcal{H}_{\infty}$ controllers. First, we use an $\mathcal{H}_{\infty}$ mixed-sensitivity design which minimizes $\bar{\sigma}(S)$ for performance, $\bar{\sigma}(T)$ for robustness and low sensitivity to noise, and $\bar{\sigma}(K S)$ to penalize large inputs. Next, we use $\mathcal{H}_{\infty}$ loop-shaping design where we specify an initial controller (plant loop shape), and apply a loop-shaping procedure that improves the robustness by maximizing the stability margin (Skogestad and Postlethwaite, 2005). The PIDF controller was used to form the initial loop shape. The results provided in this paper have been partially presented by Jahanshahi and Skogestad (2013a), and Jahanshahi et al. (2014).

This paper is organized as follows. The model identification is described in Section 2, and the new PIDF and PI tunings are introduced in Section 3. The $\mathcal{H}_{\infty}$ controller designs are presented in Section 4. Then, we verify the control solution using the small-scale and the medium-scale experiments in Section 5 and Section 6, respectively. Finally, we summarize the main conclusions and remarks in Section 7 and Section 8.

\section{Model identification}

The focus of this paper is on using an identified model for the control design. We use a mechanistic model to find a correct structure for the model to identify. In addition, we use the mechanistic model to verify the identified model in the frequency domian, because the experiments do not provide suitable data for the frequency domain analysis. First, we briefly introduce the mechanistic model. 


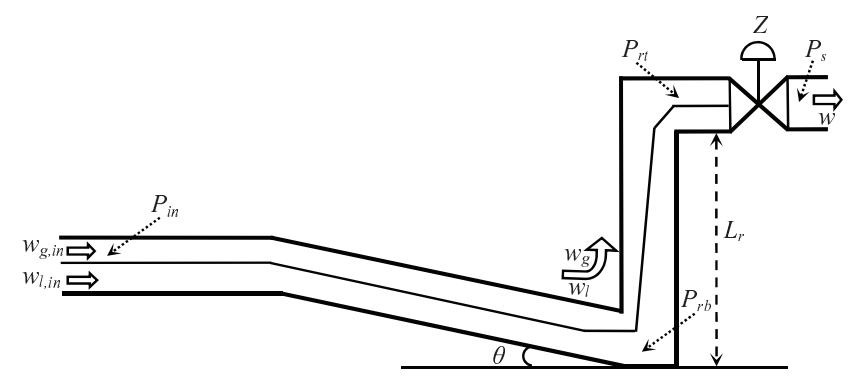

Figure 2: Schematic presentation of pipeline-riser system

\subsection{Four state mechanistic model}

Figure 2 shows a schematic presentation of the system. The inflow rates of gas and liquid to the system, $w_{g, \text { in }}$ and $w_{l, i n}$, are assumed to be independent disturbances and the top-side choke valve opening $(0<Z<100 \%)$ is the manipulated variable. A fourth-order dynamic model for this system was presented by Jahanshahi and Skogestad (2011). The state variables of this model are $m_{g p}$ (mass of gas in pipeline), $m_{l p}$ (mass of liquid in pipeline), $m_{g r}$ (mass of gas in riser) and $m_{l r}$ (mass of liquid in riser). The four state equations of the model are

$$
\begin{gathered}
\dot{m}_{g p}=w_{g, i n}-w_{g} \\
\dot{m}_{l p}=w_{l, i n}-w_{l} \\
\dot{m}_{g r}=w_{g}-\alpha w \\
\dot{m}_{l r}=w_{l}-(1-\alpha) w
\end{gathered}
$$

The flow rates of gas and liquid from the pipeline to the riser, $w_{g}$ and $w_{l}$, are determined by virtual

valve equations from the pressure drop across the riser-base. The outlet mixture flow rate, $w$, is determined by the relative opening $(Z[\%])$ of the top-side choke valve. The flow rates mentioned above and the gas mass fraction, $\alpha$, in the equations (2)-(5) are calculated by additional model equations given by Jahanshahi and Skogestad (2011).

Jahanshahi and Skogestad (2013a) found that a second-order linear model with two unstable poles and one stable zero is enough for the control design purposes. Such a model can be identified by a closed-loop step test, and this method is explained in the following. 


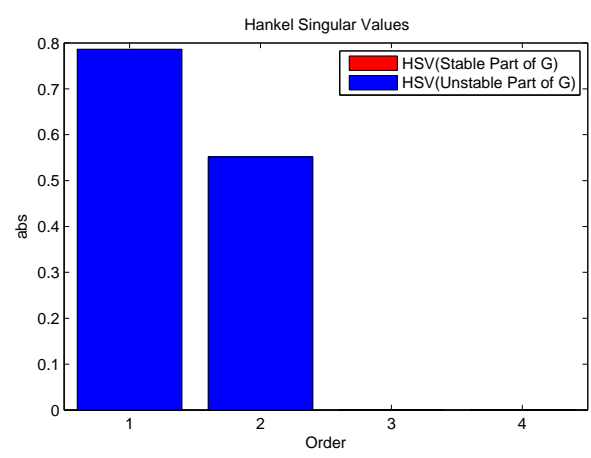

Figure 3: Hankel Singular Values of fourth order model

\subsection{Model identification from closed-loop step test}

For identification of the unstable dynamics, we need to assume a structure for the model. To get the correct model form, we linearize the four-state mechanistic model in equations (2)-(5) around the desired unstable operating point, and we get a fourth-order linear model in the form

$$
G(s)=\frac{\theta_{1}\left(s+\theta_{2}\right)\left(s+\theta_{3}\right)}{\left(s^{2}-\theta_{4} s+\theta_{5}\right)\left(s^{2}+\theta_{6} s+\theta_{7}\right)} .
$$

This model contains two unstable poles, two stable poles and two zeros. Seven parameters $\left(\theta_{i}\right)$ must be estimated to identify this model. However, if we look at the Hankel Singular Values of the fourth-order model (Figure 3), we find that the stable part of the system has little dynamic contribution. This suggests that a model with two unstable poles is sufficient for control design. Using model truncation (square root method), we obtained a reduced-order model in the form

$$
G(s)=\frac{b_{1} s+b_{0}}{s^{2}-a_{1} s+a_{0}}
$$

where $a_{0}>0$ and $a_{1}>0$. The model has two unstable poles and four parameters, $b_{1}, b_{0}, a_{1}$ and $a_{0}$, need to be estimated. If we control the unstable process in (7) using a proportional controller with gain $K_{c 0}$ (Figure 4), the closed-loop transfer function from set-point $\left(y_{s}\right)$ to output $(y)$ becomes

$$
\frac{y(s)}{y_{s}(s)}=\frac{K_{c 0}\left(b_{1} s+b_{0}\right)}{s^{2}+\left(-a_{1}+K_{c 0} b_{1}\right) s+\left(a_{0}+K_{c 0} b_{0}\right)} .
$$

This can be rewritten to the model used by Yuwana and Seborg (1982):

$$
\frac{y(s)}{y_{s}(s)}=\frac{K_{2}\left(1+\tau_{z} s\right)}{\tau^{2} s^{2}+2 \zeta \tau s+1}
$$




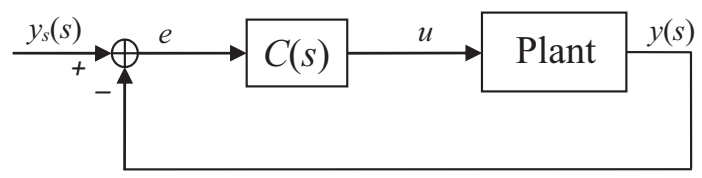

Figure 4: Closed-loop system with conventional feedback. In experimental step test we use $C(s)=K_{c 0}$.

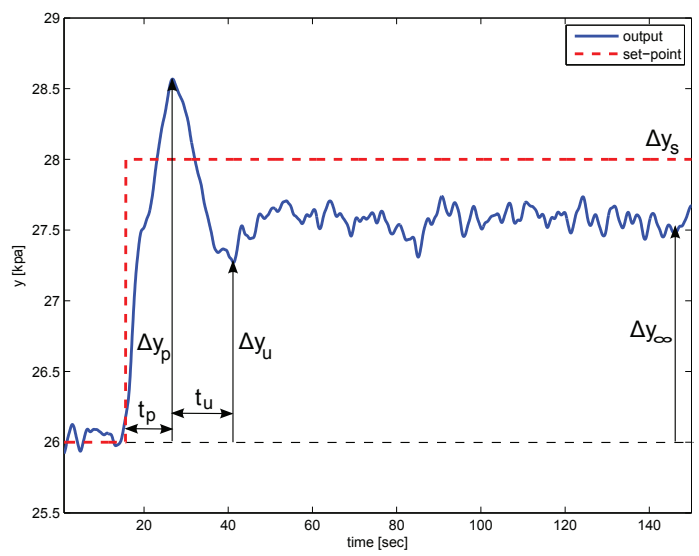

Figure 5: Experimental closed-loop step response for system stabilized with proportional control

where $K_{2}$ must be less than one as found experimentally. To estimate the four parameters $\left(K_{2}, \tau_{z}\right.$, $\tau$ and $\zeta)$ in (9), we use a very simple approach where we read six key parameters $\left(\Delta y_{p}, \Delta y_{u}, \Delta y_{\infty}\right.$,

$\Delta y_{s}, t_{p}$ and $t_{u}$ ) from the experimental closed-loop response (see Figure 5). Having the closed-loop stable model in (9), we can back-calculate the parameters of the open-loop unstable model in (7). Details are given in Appendix A.

\section{New PIDF and PI tuning based on IMC design}

\subsection{IMC design for unstable systems}

The Internal Model Control (IMC) design procedure is summarized by Morari and Zafiriou (1989). The block diagram of the IMC structure is shown in Figure 6. Here, $G(s)$ is the nominal model which in general has some mismatch with the real plant $G_{p}(s)$. $\tilde{Q}(s)$ is the inverse of the minimum phase part of $G(s)$ and $f(s)$ is a low-pass filter for robustness of the closed-loop system.

The IMC configuration in Figure 6 cannot be used directly for unstable systems; instead we use 


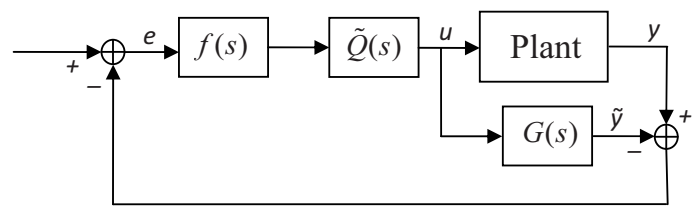

Figure 6: Block diagram of Internal Model Control system

the conventional feedback structure with the stabilizing controller

$$
C(s)=\frac{\tilde{Q}(s) f(s)}{1-G(s) \tilde{Q}(s) f(s)} .
$$

For internal stability, $\tilde{Q} f$ and $(1-G \tilde{Q} f)$ have to be stable. We use the identified model with two unstable poles and one stable zero in (7) as the plant model:

$$
G(s)=\frac{\hat{b}_{1} s+\hat{b}_{0}}{s^{2}-\hat{a}_{1} s+\hat{a}_{0}}=\frac{k^{\prime}(s+\varphi)}{\left(s-\pi_{1}\right)\left(s-\pi_{2}\right)}
$$

and we get

$$
\tilde{Q}(s)=\frac{\left(1 / k^{\prime}\right)\left(s-\pi_{1}\right)\left(s-\pi_{2}\right)}{s+\varphi}
$$

We design the filter $f(s)$ as explained by Morari and Zafiriou (1989):

$75 \quad k=$ number of RHP poles $+1=3$

$m=\max ($ number of zeros of $\tilde{Q}(s)$ - number of pole of $\tilde{Q}(s), 1)=1$ (to make $Q=\tilde{Q} f$ proper)

$n=m+k-1=3$ (filter order)

With $n=3$, the filter is in the following from:

$$
f(s)=\frac{\alpha_{2} s^{2}+\alpha_{1} s+\alpha_{0}}{(\lambda s+1)^{3}}
$$

where $\lambda$ is the adjustable closed-loop time-constant. We choose $\alpha_{0}=1$ to get integral action and the coefficients $\alpha_{1}$ and $\alpha_{2}$ are calculated by solving the following system of linear equations:

$$
\left(\begin{array}{lll}
\pi_{1}{ }^{2} & \pi_{1} & 1 \\
\pi_{2}{ }^{2} & \pi_{2} & 1
\end{array}\right)\left(\begin{array}{l}
\alpha_{2} \\
\alpha_{1} \\
\alpha_{0}
\end{array}\right)=\left(\begin{array}{c}
\left(\lambda \pi_{1}+1\right)^{3} \\
\left(\lambda \pi_{2}+1\right)^{3}
\end{array}\right)
$$

Finally, from (10) the feedback version of the IMC controller becomes

$$
C(s)=\frac{\left[\frac{1}{k^{\prime} \lambda^{3}}\right]\left(\alpha_{2} s^{2}+\alpha_{1} s+1\right)}{s(s+\varphi)} .
$$




\subsection{PIDF implementation of IMC controller}

Here, we obtain PIDF settings from the proposed IMC controller. The IMC controller in (15) is a second order transfer function which can be written in form of a PID controller with a low-pass filter.

$$
K_{P I D F}(s)=K_{c}\left(1+\frac{1}{s T_{i}}+\frac{T_{d} s}{T_{f} s+1}\right)
$$

where

$$
\begin{gathered}
T_{f}=1 / \varphi \\
T_{i}=\alpha_{1}-T_{f} \\
K_{c}=\frac{T_{i} T_{f}}{k^{\prime} \lambda^{3}} \\
T_{d}=\frac{\alpha_{2}}{T_{i}}-T_{f}
\end{gathered}
$$

For the controller work in practice, we require that $K_{c}<0$ and $T_{d}>0$; and we must choose $\lambda$ such

that these two conditions are satisfied. This was observed in the experiments.

\subsection{PI-controller tuning}

Next, we consider PI control. There are many approaches to get tuning values for PI control. For example, relay-feedback auto-tuning has been used by Ogazi et al. (2009) for PI tuning based on a first-order unstable model. Here, we obtain the PI tuning based on the IMC controller from the previous Section. We consider a PI controller in the following form

$$
K_{P I}(s)=K_{c}\left(1+\frac{1}{\tau_{I} s}\right),
$$

The PIDF controller in (16) can be approximated by a PI-controller by considering the high- and low-frequency asymptotes of $C(s)$ in (15).

$$
\begin{gathered}
K_{c}=\lim _{s \rightarrow \infty} C(s)=\frac{\alpha_{2}}{k^{\prime} \lambda^{3}} \\
\tau_{I}=\frac{K_{c}}{\lim _{s \rightarrow 0} s C(s)}=\alpha_{2} \varphi
\end{gathered}
$$




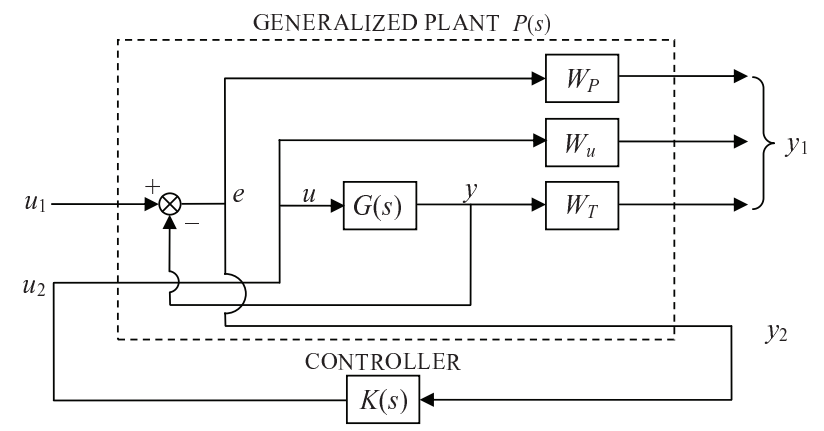

Figure 7: Closed-loop system for mixed sensitivity control design

\section{4. $\mathcal{H}_{\infty}$ control based on identified model}

\section{1. $\mathcal{H}_{\infty}$ mixed-sensitivity design}

We consider an $\mathcal{H}_{\infty}$ problem where we want to bound $\bar{\sigma}(S)$ for performance, $\bar{\sigma}(T)$ for robustness and low sensitivity to noise, and $\bar{\sigma}(K S)$ to penalize large inputs. These requirements may be combined into a stacked $\mathcal{H}_{\infty}$ problem (Skogestad and Postlethwaite, 2005).

$$
\min _{K}\|N(K)\|_{\infty}, \quad N \triangleq\left[\begin{array}{c}
W_{u} K S \\
W_{T} T \\
W_{P} S
\end{array}\right]
$$

where $W_{u}, W_{T}$ and $W_{P}$ determine the desired shapes of $K S, T$ and $S$, respectively. Typically, $W_{P}^{-1}$ is chosen to be small at low frequencies to achieve good disturbance attenuation (i.e., performance), and $W_{T}^{-1}$ is chosen to be small outside the control bandwidth, which helps to ensure good stability margin (i.e., robustness). $W_{u}$ is often chosen as a constant. The solution to this optimization problem gives a stabilizing controller $K$ that satisfies (Doyle et al., 1989; Glover and Doyle, 1988):

$$
\begin{aligned}
\bar{\sigma}(K S(j \omega)) & \leq \gamma \underline{\sigma}\left(W_{u}^{-1}(j \omega)\right) \\
\bar{\sigma}(T(j \omega)) & \leq \gamma \underline{\sigma}\left(W_{T}^{-1}(j \omega)\right) \\
\bar{\sigma}(S(j \omega)) & \leq \gamma \underline{\sigma}\left(W_{P}^{-1}(j \omega)\right) .
\end{aligned}
$$

$y_{2}$ is the particular output for feedback control in the generalized plant in Figure 7. The value of

${ }_{85} \gamma$ in equation (25) should be as small as possible for good controllability. However, it depends on the design specifications $W_{u}, W_{T}$ and $W_{P}$. 


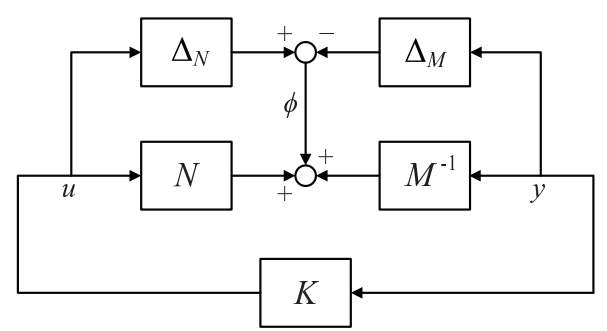

Figure 8: $\mathcal{H}_{\infty}$ robust stabilization problem

\section{2. $\mathcal{H}_{\infty}$ loop-shaping design}

We consider the stabilization of the plant $G$ which has a normalized left coprime factorization

$$
G=M^{-1} N
$$

where we have dropped the subscripts from $M$ and $N$ for simplicity. A perturbed plant model $G_{p}$ can then be written as

$$
G_{p}=\left(M+\Delta_{M}\right)^{-1}\left(N+\Delta_{N}\right)
$$

where $\Delta_{M}$ and $\Delta_{N}$ are stable unknown transfer functions which represent the uncertainty in the nominal plant model $G$. The objective of robust stabilization is to stabilize not only the nominal model $G$, but a family of perturbed plants defined by

$$
G_{p}=\left\{\left(M+\Delta_{M}\right)^{-1}\left(N+\Delta_{N}\right):\left\|\left[\Delta_{N} \Delta_{M}\right]\right\|_{\infty}<\epsilon\right\}
$$

where $\epsilon>0$ is then the stability margin (Skogestad and Postlethwaite, 2005). To maximize this stability margin is the problem of robust stabilization of normalized coprime factor plant description as introduced and solved by Glover and McFarlane (1989).

For the perturbed feedback system of Figure 8, the stability property is robust if and only if the nominal feedback system is stable and

$$
\gamma_{K} \triangleq\left\|\left[\begin{array}{c}
K \\
I
\end{array}\right](I-G K)^{-1} M^{-1}\right\|_{\infty} \leq \frac{1}{\epsilon}
$$

Notice that $\gamma_{K}$ is the $\mathcal{H}_{\infty}$ norm from $\phi$ to $\left[\begin{array}{l}u \\ y\end{array}\right]$ and $(I-G K)^{-1}$ is the sensivity function for this positive feedback arragmenet. A small $\gamma_{K}$ is corresponding to a large stability margin. 


\section{Small-scale experiments}

\subsection{Exprimental setup}

The experiments were performed on a small-scale laboratory rig for anti-slug control at the Chemical Engineering Department of NTNU. Figure 9 shows a schematic presentation of the laboratory setup. The pipeline and the riser are made from flexible pipes with $2 \mathrm{~cm}$ inner diameter. The length of the pipeline is $4 \mathrm{~m}$, and it is inclined with a $15^{\circ}$ angle. The height of the riser is 3 $m$. A buffer tank is used to simulate the effect of a long pipe with the same volume, such that the total resulting length of pipe would be about $70 \mathrm{~m}$.

The topside choke valve opening $Z$ is used as the input for control $(M V=Z)$. The separator pressure after the topside choke valve is nominally constant at atmospheric pressure. The feed into the pipeline is assumed to be at constant flow rates, 4 litre/min of water and 4.5 litre/min of air. With these boundary conditions, the critical valve opening where the system switches from stable (non-slug) to oscillatory (slug) flow is at $Z^{*}=15 \%$ for the top-side valve. The bifurcation diagram is shown in Figure 10 .

The desired steady-state (dashed middle line) at slugging conditions $(Z>15 \%)$ is unstable, but it can be stabilized using feedback control. The controlled output throughout this paper is the pressure at the inlet of the pipeline $\left(C V=P_{i n}\right)^{1}$. The slope of the steady-state line (in the middle) is the static gain of the system, $k=\partial y / \partial u=\partial P_{i n} / \partial Z$. As the valve opening increase this slope decreases, and the gain finally approaches zero. This makes control of the system with large valve openings very difficult. On the other hand, large valve openings are desirable because this minimizes the pressure drop over the valve and increases the production rate.

As mentioned above stabilizing the system at large valve openings (low pressure set-points) is difficult because of the small gain. We decrease the controller set-point to see if the controller can stabilize the system with a lower set-point. We use the same set of descending pressure setpoints in all experiments. The controllers are tuned (designed) for a valve opening of $Z=30 \%$, and controllers with good gain margin can stabilize the system with larger valve openings (lower set-points). Note that the system has a smaller gain at $\mathrm{Z}=30 \%$ compared to $\mathrm{Z}=20 \%$, hence, a

\footnotetext{
${ }^{1}$ As shown in Figure 9, we use the buffer tank pressure $P_{1}$ instead of the mixing point pressure $P_{3}$ as the inlet pressure which is the controlled output $\left(C V=P_{i n}\right)$ in the experiments. Actually, two pressures are very close, only the buffer thank pressure is less noisy, because the air filters out the noise naturally.
} 


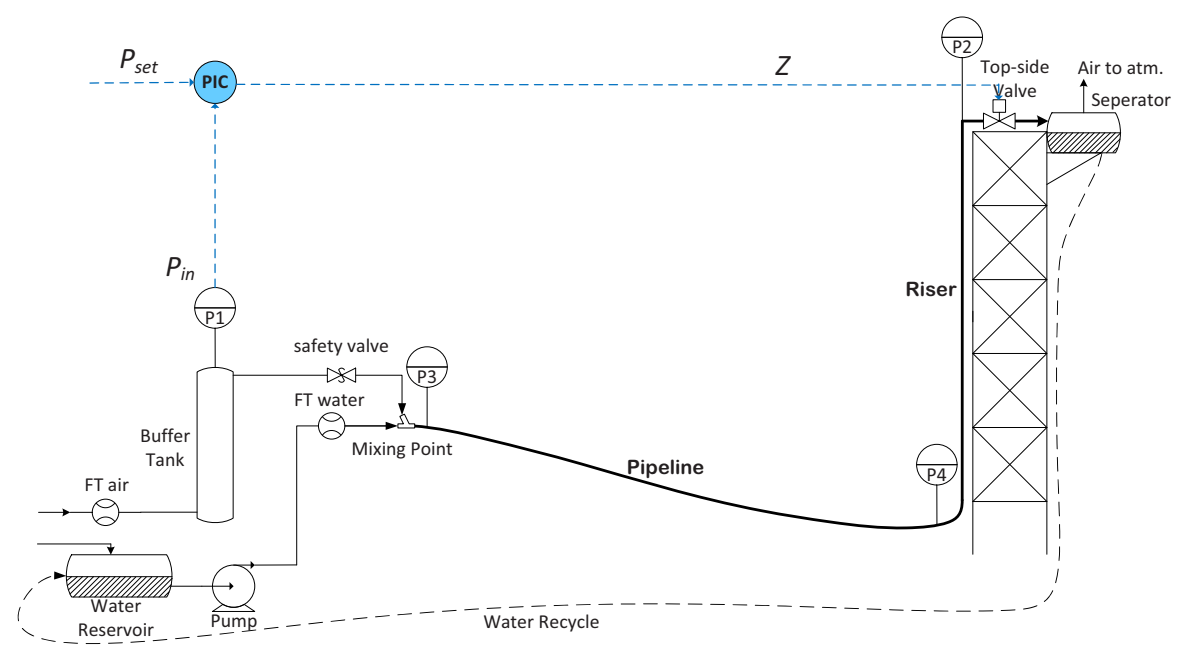

Figure 9: Small-scale experimental rig

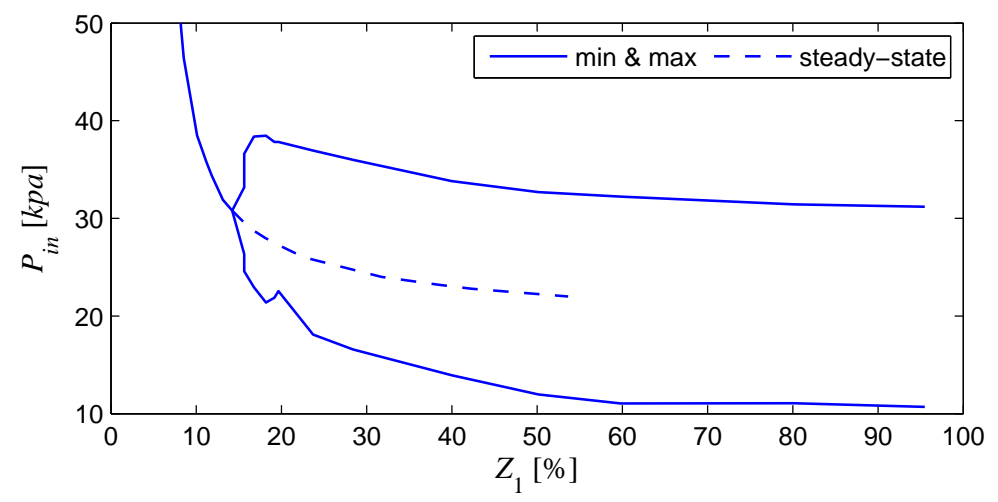

Figure 10: Experimental bifurcation diagrams for small-scale rig 
want to stabilize the system with large valve openings up to $\mathrm{Z}=50 \%$, while the controller designed for $\mathrm{Z}=20 \%$ was not able to stabilize the system at large valve openings.

To have an impartial comparison for robustness of the controllers, we tune the controllers such that all of them result in the same input usage $\left(M_{k s}=50\right)$. Here, $M_{k s}$ is peak of $K S$ and for the PIDF controller in (16) it becomes

$$
M_{k s}=-K_{c}\left(T_{d} / T_{f}+1\right)
$$

\subsection{Model identification}

Valve opening of $Z=20 \%$

The flow regime switches to slugging flow at a valve opening of $Z=15 \%$, hence it is unstable at $Z=20 \%$. We closed the loop with a proportional controller with $K_{c 0}=-10$, and changed the set-point by $2 \mathrm{kPa}$ (Figure 11). Since the response is noisy, a low-pass filter was used to reduce the noise effect. Then, we use the method described in Section 2.2 to identify the closed-loop stable transfer function:

$$
\frac{y(s)}{y_{s}(s)}=\frac{3.13 s+0.81}{20.62 s^{2}+2.20 s+1}
$$

The identified closed-loop transfer function is shown by the red line in Figure 11. From this, we back-calculate to an open-loop unstable process model:

$$
G(s)=\frac{-0.015(s+0.26)}{s^{2}-0.045 s+0.0093}
$$

If we linearize the four-state mechanistic model given in (2)-(5) at the operating point $Z=20 \%$, we get the following fourth-order model.

$$
G(s)=\frac{-0.28(s+20.21)(s+0.27)}{\left(s^{2}-0.046 s+0.013\right)\left(s^{2}+21.68 s+256.1\right)}
$$

The frequency response of the identified model at the valve opening $Z=20 \%$ (32) is compared to the mechanistic model (33) in Figure 12. This agreement is surprisingly good. The two unstable poles of the mechanistic model are $p=0.0233 \pm 0.1096 i$, and the unstable poles of the identified model are $p=0.0227 \pm 0.0937 i$. 


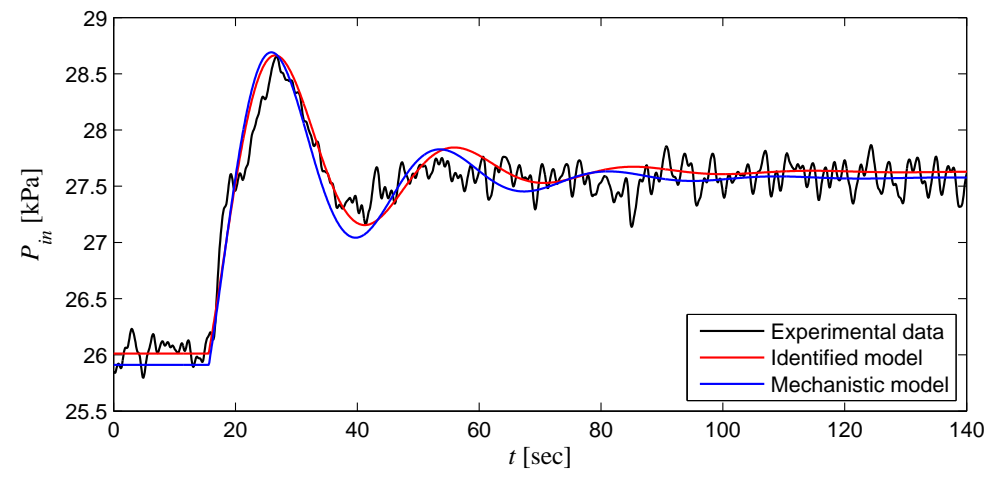

Figure 11: Experimental closed-loop step test compared with identified model and mechanistic model $(Z=20 \%)$
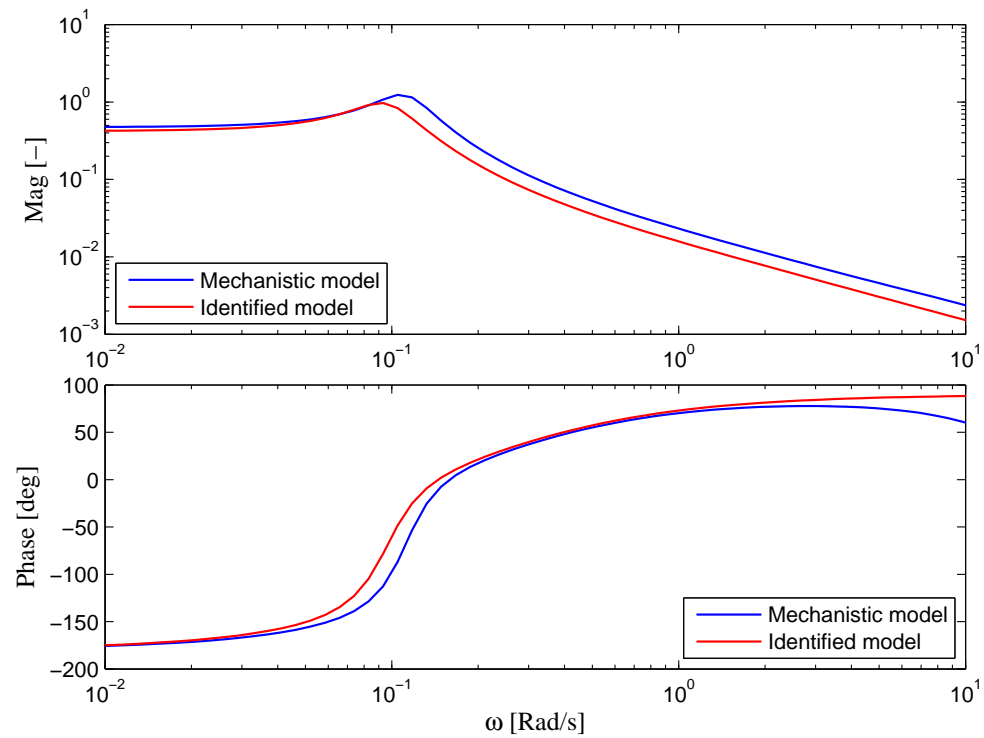

Figure 12: Comparison of identified and mechanistic models in frequency domain $(Z=20 \%)$ 


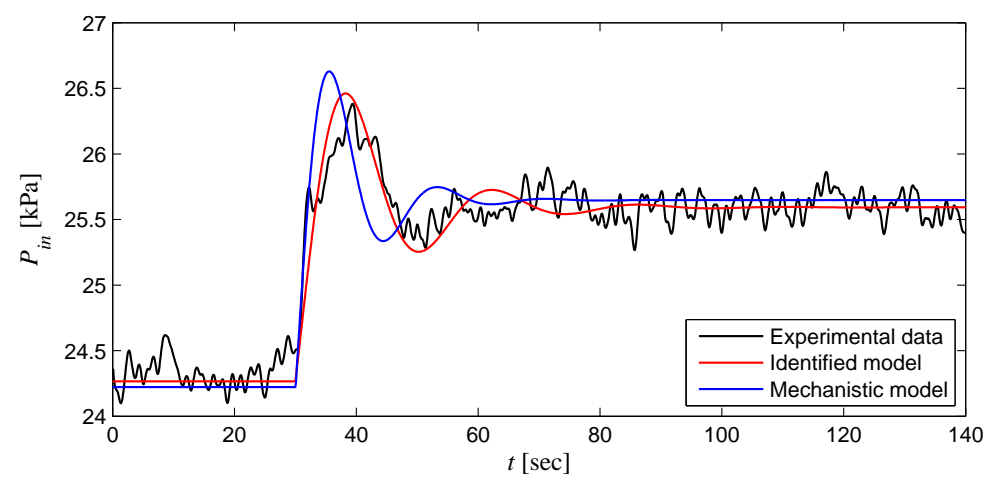

Figure 13: Experimental closed-loop step test compared with identified model and mechanistic model $(Z=30 \%)$

Valve opening of $Z=30 \%$

We repeated the previous experiment at $Z=30 \%$ valve opening. We closed the loop using a proportional controller with $K_{c 0}=-20$ and changed the set-point by $2 k P a$ (Figure 13). Then, we use the method explained in Section 2.2 to identify the closed-loop stable transfer function:

$$
\frac{y(s)}{y_{s}(s)}=\frac{2.634 s+0.6635}{13.39 s^{2}+2.097 s+1}
$$

The identified closed-loop transfer function is shown by the red line in Figure 13. Then, we backcalculate to an open-loop unstable system:

$$
G(s)=\frac{-0.0098(s+0.25)}{s^{2}-0.04 s+0.025}
$$

The four-state mechanistic model given in (2)-(5), linearized at the operating point $Z=30 \%$, results in the following fourth-order model.

$$
G(s)=\frac{-0.18(s+20.18)(s+0.27)}{\left(s^{2}-0.17 s+0.023\right)\left(s^{2}+26.57 s+303.4\right)}
$$

The frequency response of the identified model at the valve opening $Z=30 \%$ (35) is compared to the mechanistic model (36) in Figure 14. The agreement is very good also in this case. The two unstable poles of the mechanistic model are $p=0.0860 \pm 0.1235 i$, and the two poles of the identified model are $p=0.0200 \pm 0.1572 i$.

Comparing Figure 11 and Figure 13, we see that the mechanistic model matches the experiments and the identified model well for $Z=20 \%$, but it does not mach very well for $Z=30 \%$. Taking 

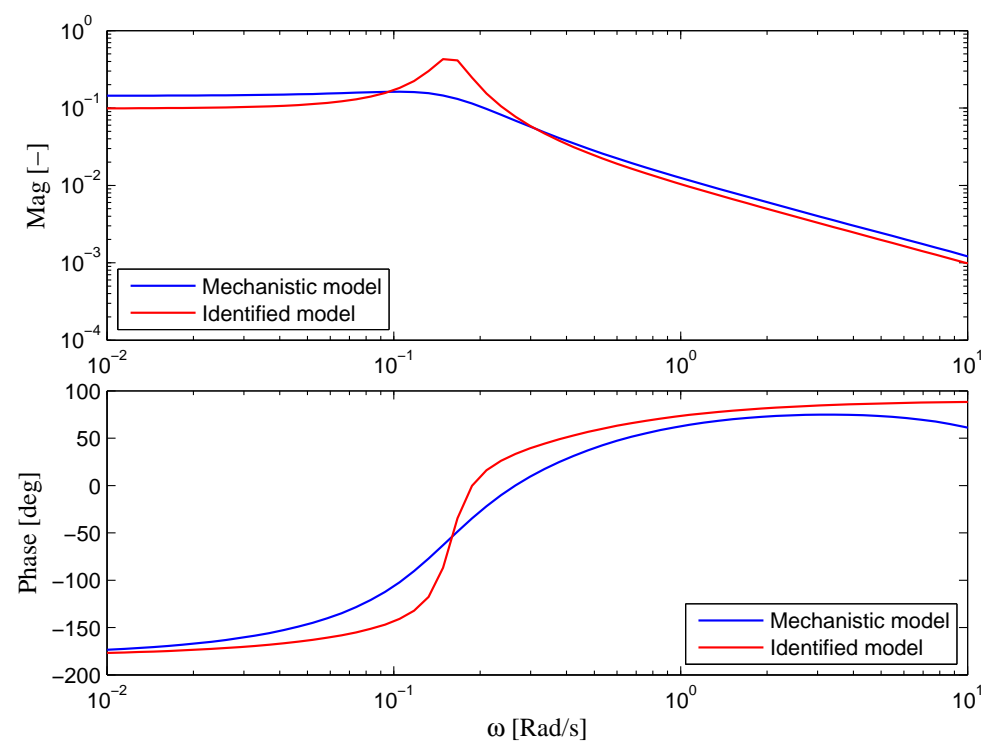

Figure 14: Comparison of identified and mechanistic models in frequency domain $(Z=30 \%)$

into account that the mechanistic model is very simplified, and it has been fitted to the open-loop data, not the closed-loop test data, its agrement for the closed-loop test is very good. In the tuning procedure of the mechanistic model (Jahanshahi and Skogestad, 2011), the focus was on the frequency at the onset of the slugging ( $Z=15 \%$ for this case). We observe that the mechanistic model at $Z=20 \%$ is more correct compared to $Z=30 \%$, because $Z=20 \%$ is closer to the onset of the slugging.

\subsection{IMC (PIDF)}

We used the identified model in (35) for an IMC design. We chose the filter time constant $\lambda=6.666 s$ to get $M_{k s}=50$. The resulting IMC controller becomes

$$
C(s)=\frac{-50\left(s^{2}+0.0867 s+0.0069\right)}{s(s+0.25)} .
$$

Note that the controller has complex zeros. The corresponding PIDF setting values are $K_{c}=$ $-11.84, T_{i}=8.59 \mathrm{~s}, T_{d}=12.89 \mathrm{~s}$ and $T_{f}=4 \mathrm{~s}$. Figure 15 shows performance of the PIDF controller larger time delay. 

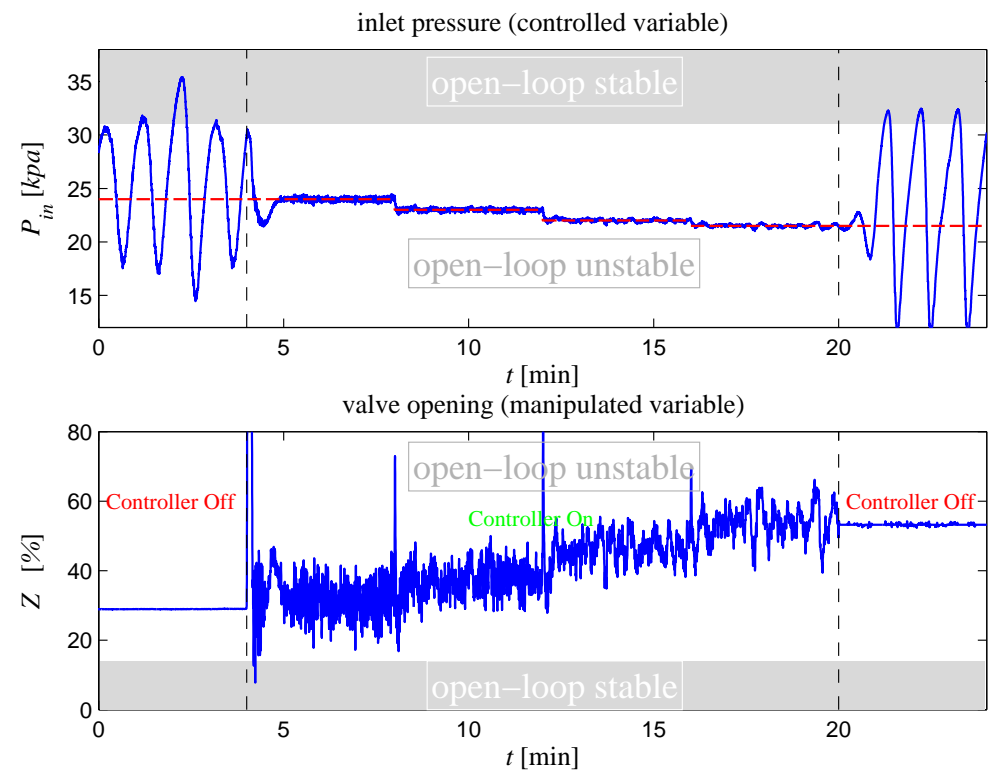

Figure 15: Experimental result of PIDF with $K_{c}=-11.84, T_{i}=8.59 \mathrm{~s}, T_{d}=12.89 \mathrm{~s}$ and $T_{f}=4 \mathrm{~s}$

In general, the filter time constant $\lambda$ for the IMC design must be related to the frequency of the system dynamics. For the slugging case, we found a rule of thumb that $\lambda \approx 0.1 \times T$ where $T$ is the period of the slugging oscillations. The period of slugging for the small-scale experimental rig is around $T=68 \mathrm{sec}$ and for the medium-scale rig is around $T=200 \mathrm{sec}$.

\subsection{PI tuning}

Next, we obtain the PI tuning from the IMC controller (37) as explained in Section 3.3. The PI tuning parameters are $K_{c}=-50.00$ and $\tau_{I}=36.30 \mathrm{~s}$. Figure 16 shows result of experiment using the PI controller. This controller was stable with 1 sec added time delay.

We used the IMC controller (37) to obtain the initially shaped plant for the $\mathcal{H}_{\infty}$ loop-shaping design. The following fifth-order controller was resulted.

$$
C(s)=\frac{-188.49\left(s^{2}+0.02 s+0.005\right)\left(s^{2}+0.087 s+0.0069\right)}{s(s+0.25)(s+3.76)\left(s^{2}+0.082 s+0.0067\right)}
$$

The experimental result of the controller in (38) is shown in Figure 17. The closed-loop system was stable with $2 \mathrm{sec}$ added time delay; the system becomes unstable for any larger time delay. 

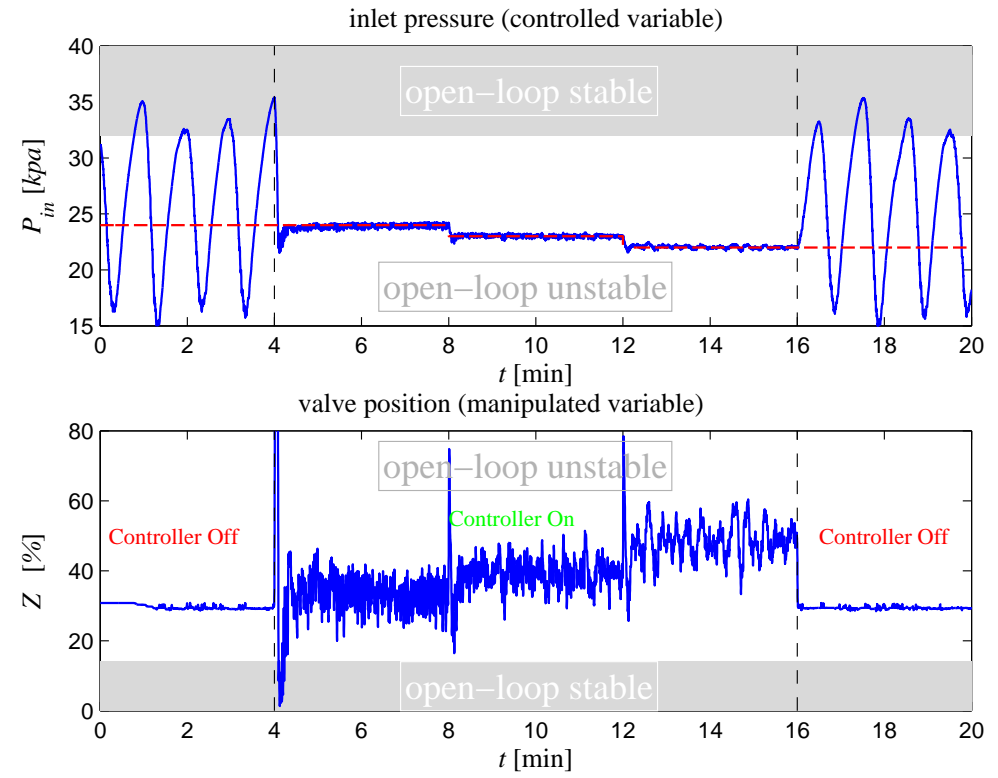

Figure 16: Experimental result of PI controller with $K_{c}=-50.00, \tau_{I}=36.30 \mathrm{~s}$
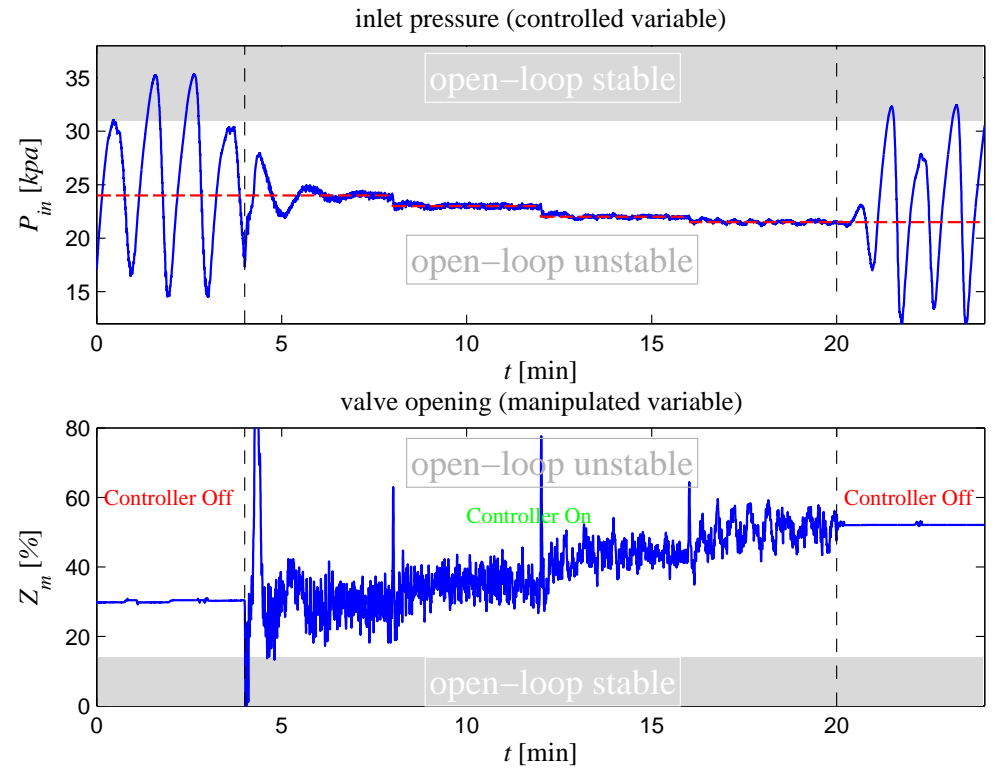

Figure 17: Experimental result of loop-shaping $\mathcal{H}_{\infty}$ 

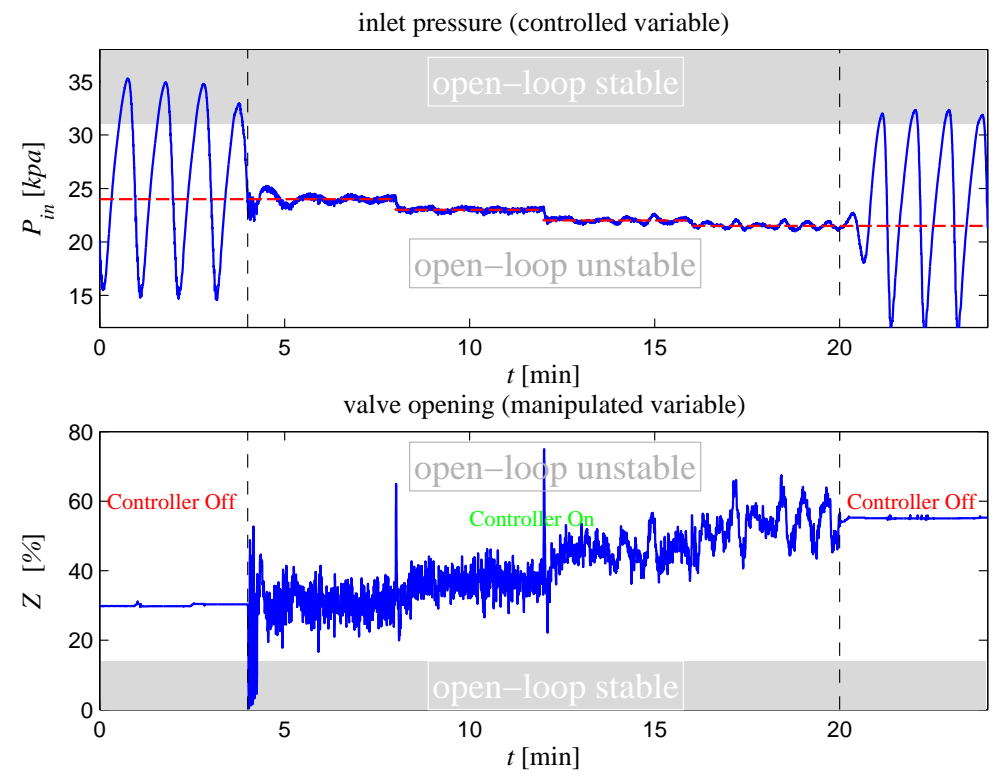

Figure 18: Experimental result of mixed-sensitivity $\mathcal{H}_{\infty}$

\section{6. $\mathcal{H}_{\infty}$ mixed-sensitivity}

We design the $\mathcal{H}_{\infty}$ mixed-sensitivity controller with the following design specifications:

$$
\begin{gathered}
W_{P}(s)=\frac{s / M_{s}+\omega_{B}}{s+\omega_{B} A}, \\
W_{T}(s)=\frac{s /\left(10 \omega_{B}\right)+1}{0.01 s+1}, \\
W_{u}=0.0135,
\end{gathered}
$$

where $M_{s}=1, \omega_{B}=0.14$ and $A=0.01$. We chose these design specifications so that we achieve $M_{k s}=50$ and good robustness properties. We get the following fourth-order stabilizing controller.

$$
C(s)=\frac{-9.08 \times 10^{6}(s+100)\left(s^{2}+0.0137 s+0.011\right)}{\left(s+1.8 \times 10^{5}\right)(s+112.5)(s+0.231)(s+0.0014)}
$$

We achieved $\gamma=1.21$ with this controller; the experimental performance is shown in Figure 18.

This controller was stable with 2 sec added time delay to the output. 


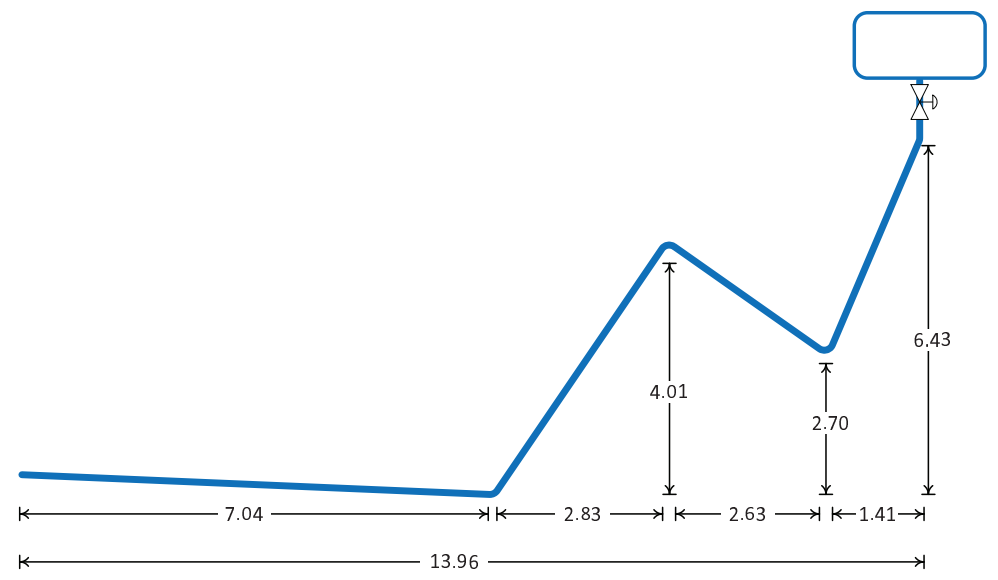

Figure 19: Experimental setup for medium-scale S-riser rig, all dimensions in meter

\section{Medium-scale experiments}

\subsection{Experimental setup}

The tuning procedures were validated also on a medium-scale test rig. This test rig is an S-riser with a height of about $7 \mathrm{~m}$. Other dimensions of this experimental set-up are shown in Figure 19. This riser is made from stainless steel pipes with inner diameter of $50 \mathrm{~mm}$. Similar to the small scale setup, an air buffer thank is installed at inlet to emulate the effect of a long pipeline with the same volume. The volume of the buffer tank is 200 litres; this is equivalent $101.86 \mathrm{~m}$ of pipe. The inlet flow rates to the system are $0.0024 \mathrm{~kg} / \mathrm{sec}$ air and $0.3927 \mathrm{~kg} / \mathrm{sec}$ water. The outlet separator pressure is constant at the atmospheric pressure. With these boundary conditions, the system switches from non-slug to slugging flow conditions at $Z^{*}=16 \%$ opening of the topside valve.

\subsection{IMC (PIDF) controller at $Z=18 \%$}

Figure 20 shows a closed-loop step test performed on the S-riser. A proportional controller with the gain $K_{c 0}=-250$ was used for the test. The pressure set-point before the step test was 155 $k P a$ which results in a valve opening of $Z=18 \%$ (region of unstable open-loop operation). We identified an unstable model as the following:

$$
G(s)=\frac{-5.6 \times 10^{-4}(s+0.082)}{s^{2}-0.069 s+0.0040}
$$




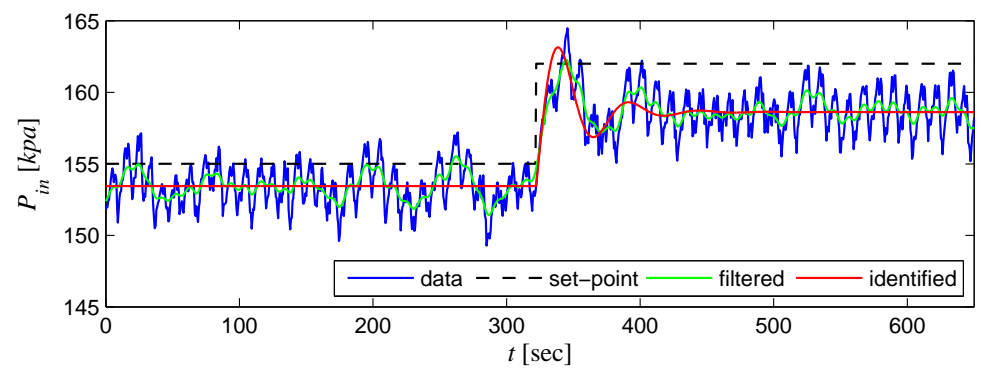

Figure 20: Closed-loop step test on medium-scale rig

By choosing $\lambda=24.5$, we designed the following IMC controller:

$$
C(s)=\frac{-340.75\left(s^{2}+0.0052 s+0.00036\right)}{\mathrm{s}(\mathrm{s}+0.0816)}
$$

The corresponding PIDF tuning are $K_{c}=-3.47, T_{i}=2.33 s, T_{d}=1.19 \times 10^{4} s$ and $T_{f}=12.25 s$. Experimental result of control using this PIDF tuning is shown in Figure 21. In this experiment, we decreased the set-point until the system becomes unstable. This controller was able to control the system up to a $Z=32 \%$ valve opening, which is two time of the critical valve opening $Z^{*}=16 \%$.

\subsection{PI-controller tuning at $Z=18 \%$}

The PI tuning obtained from the IMC controller in (44) are $K_{c}=-340.75$ and $\tau_{I}=229.23 \mathrm{~s}$. The experimental result is given in Figure 22 where system was stabilized up to $Z=24 \%$.

The controller loses the stability from a certain position of the valve position, as shown in Figure 21 and Figure 22. This happens because the gain of the system decreases drastically for the lower set-points due to nonlinearity, but the controller gain is still the same. A gain scheduling scheme can be used to stabilize the system with larger valve openings. Figure 21 and Figure 22 show the valve position is oscillating while the system is open-loop. Actually, this is the controller output (the controller was in auto mode) but the controller is not controlling the valve.

\section{Discussion}

Unlike the medium-scale experimental setup, the small-scale experimental setup was very easy to start up and load different controllers from Matlab. Therefore, we were able to test more controllers using the small-scale experiments. Performance and robustness of the different controllers used 

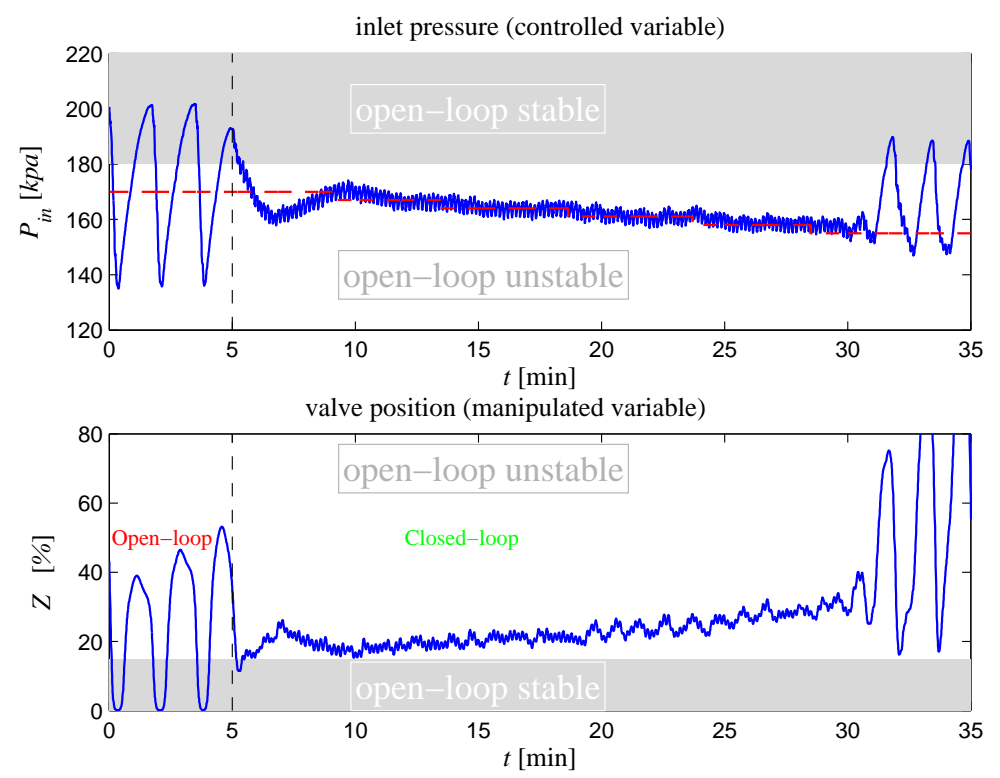

Figure 21: Experimental result of PIDF controller with $K_{c}=-3.47, T_{i}=2.33 \mathrm{~s}, T_{d}=1.19 \times 10^{4} s$ and $T_{f}=12.25 s$ on medium-scale rig
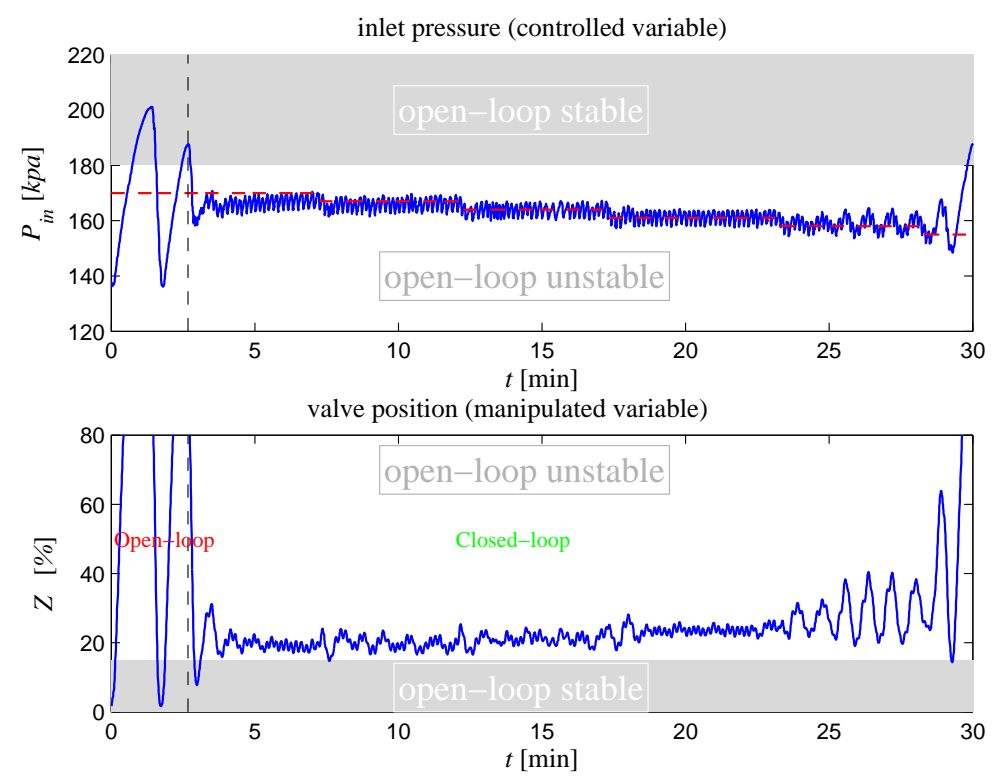

Figure 22: Experimental result of PI controller with $K_{c}=-340.75$ and $\tau_{I}=229.23 \mathrm{~s}$ on medium-scale rig 
Table 1: Comparison of different controllers in small-scale experiments

\begin{tabular}{|c|c|c||c|c|c|c|c|}
\hline Controller & $Z_{\max }$ & ISE & $\|S\|_{\infty}$ & $\|T\|_{\infty}$ & $\|K S\|_{\infty}$ & GM & DM \\
\hline $\mathcal{H}_{\infty}$ Loop Shaping & $55 \%$ & 184.98 & 1.10 & 1.12 & 50 & 0.10 & 2.48 \\
IMC PIDF & $55 \%$ & 171.45 & 1.00 & 1.19 & 50 & 0.11 & 2.49 \\
IMC PI & $50 \%$ & 178.03 & 1.03 & 1.38 & 51.64 & 0.10 & 1.80 \\
$\mathcal{H}_{\infty}$ Mixed Sensitivity & $50 \%$ & 330.25 & 1.00 & 1.18 & 50 & 0.15 & 3.00 \\
\hline
\end{tabular}

in the small-scale experiments are compared in Table 1. We use two performance measures for

the controllers, the Integral Square Error (ISE) and the maximum valve opening $Z_{\max }$ that each controller can stabilize the system. The robustness is evaluated based on peak of $T$, gain-margin and delay-margin. The theoretical delay margin (DM) values given in Table 1 are not exactly the same as what we found in the experiments, but the values are consistent comparing different controllers.

The $\mathcal{H}_{\infty}$ loop-shaping controller has the best gain-margin, and the smallest value for the peak of $T$. The $\mathcal{H}_{\infty}$ loop-shaping and the PIDT can stabilize the system up to a similar valve opening around $55 \%$. However, the $\mathcal{H}_{\infty}$ loop-shaping controller is more robust and shows less oscillations at the last pressure set-point, compared to the PIDF. The PIDF controller shows the best ISE, and the PI controller is the second best controller for ISE.

The $\mathcal{H}_{\infty}$ mixed-sensitivity controller shows a large value for ISE, because for the lower set-points it has some oscillations (see Figure 18); this is due to the poor gain-margin of the controller. The $\mathcal{H}_{\infty}$ mixed-sensitivity has the largest (worst) value for the gain-margin, but the best delay-margin.

In summary, considering combined performance and robustness measures, the $\mathcal{H}_{\infty}$ loop-shaping controller is the best, and the PIDF controller is the second one in our results. Note that we used the IMC controller as the initial controller for the loop-shaping design and the improvement of the controller is obvious.

\section{Conclusion}

In this paper we developed and compared feedback controllers for unstable multiphase flow in risers. The study included simple PIDF tuning rules, PI-tuning and two $\mathcal{H}_{\infty}$ controllers. The comparison was based on experimental tests carried out in two prototype flow systems. 
We identified a second-order unstable model for the system, and compared the identified model with a mechanistic model in time domain and the frequency domain. The identified model for a valve opening of $20 \%$ is very close to the mechanistic model. Also, agreement between the models for a valve opening of $30 \%$ was good.

We showed that for this case performance and robustness of a PIDF controller is close to $\mathcal{H}_{\infty}$ controllers (see Table 1). Slightly better results can be achieved by the $\mathcal{H}_{\infty}$ loop-shaping approach, where we employ the PIDF controller to obtain the initially shaped plant. However, this method results in higher order controllers which may not be desired by the practitioners.

The $\mathcal{H}_{\infty}$ mixed-sensitivity design is more involved as it requires tuning of many weights simultaneously. However, we could not achieve better results than that of a PIDF controller for this case and further investigation is needed.

We tested the PIDF and PI controllers on a medium-scale flow system which shows applicability of the proposed tuning rules on large systems. Testing the tuning rules in real applications is recommended. A potential issues when going from medium scale to proper offshore installation is that the top-side choke valve in real application is slower than what we have used in the experiments.

The PID and PI are the most common controllers used at the offshore oilfields (e.g. CA Function Block in PLCs), and gain-scheduling of multiple linear controllers is a common approach to include the system nonlinearity. Considering that the pipeline-riser system is highly nonlinear, gain-scheduling can increase the operation range of the controller (i.e. a wider range of the valve opening). This solution has been tested in another publication of the authors (Jahanshahi and Skogestad, 2013b). Therefore, we believe that the proposed PIDF controller or a gain-scheduling of PIDF controllers is an effective industry-ready solution for the slugging problem. However, the $\mathcal{H}_{\infty}$ controllers also can be used in a gain-scheduling scheme if the control system can handle higher order controllers.

\section{Acknowledgements}

Funding for this research was provided by SIEMENS, Oil and Gas Solutions. The medium-scale experiment were carried out at the multi-phase laboratory, Department of Energy and Process Engineering (EPT). We would like to thank professor Ole Jørgen Nydal for providing us this opportunity. We also acknowledge help from Mahnaz Esmaeilpour, the master student who carried out the medium-scale experiments. 


\section{References}

Courbot, A. (1996). Prevention of severe slugging in the dunbar 16 inches multiphase pipeline. In Proceedings of the Annual Offshore Technology Conference, volume 4, 445-452. SPE no. 8196.

Di Meglio, F., Kaasa, G.O., Petit, N., and Alstad, V. (2010). Model-based control of slugging flow: an experimental case study. In American Control Conference, 2995-3002. Baltimore, USA.

Doyle, J., Glover, K., Khargonekar, P., and Francis, B. (1989). State-space solutions to standard $\mathcal{H}_{2}$ and $\mathcal{H}_{\infty}$ control problems. IEEE Transactions on Automatic Control, 34(8), 831-847.

Glover, K. and Doyle, J.C. (1988). State-space formulae for all stabilizing controllers that satisfy an $\mathcal{H}_{\infty}$-norm bound and relations to relations to risk sensitivity. Systems and Control Letters, 11(3), 167-172.

Glover, K. and McFarlane, D. (1989). Robust stabilization of normalized coprime factor plant descriptions with $h_{\infty}$-bounded uncertainty. IEEE Transactions on Automatic Control, 34(8), $821-830$.

Godhavn, J.M., Fard, M.P., and Fuchs, P.H. (2005). New slug control strategies, tuning rules and experimental results. Journal of Process Control, 15, 547-557.

Havre, K., Stornes, K., and Stray, H. (2000). Taming slug flow in pipelines. ABB Review, 4, 55-63.

Jahanshahi, E. and Skogestad, S. (2011). Simplified dynamical models for control of severe slugging in multiphase risers. In 18th IFAC World Congress, 1634-1639. Milan, Italy.

Jahanshahi, E., de Oliveira, V., Gimholt, C., and Skogestad, S. (2014). A comparison between internal model control pidf, optimal pidf and robust controllers for unstable flow in risers. In 19th IFAC World Congress. Cape Town, South Africa.

Jahanshahi, E. and Skogestad, S. (2013a). Closed-loop model identification and pid/pi tuning for robust anti-slug control. In 10th IFAC International Symposium on Dynamics and Control of Process Systems (DyCOPS), 233-240. Mumbai, India.

Jahanshahi, E. and Skogestad, S. (2013b). Comparison between nonlinear modelbased controllers and gain-scheduling internal model control based on identified model. In 52nd IEEE Conference on Decision and Control. Florence, Italy. 
Morari, M. and Zafiriou, E. (1989). Robust Process Control. Prentice Hall, Englewood Cliffs, New Jersey.

Ogazi, A., Ogunkolade, S., Cao, Y., Lao, L., and Yeung, H. (2009). Severe slugging control through open loop unstable pid tuning to increase oil production. 17-32.

Skogestad, S. and Postlethwaite, I. (2005). Multivariable Feedback Control: Analysis and Design. Wiley \& Sons, Chichester, West Sussex, UK.

Yocum, B. (1973). Offshore riser slug flow avoidance: Mathematical models for design and optimization. In SPE European Meeting, SPE no. 4312-MS. Society of Petroleum Engineers, London, United Kingdom.

Yuwana, M. and Seborg, D.E. (1982). A new method for on-line controller tuning. AIChE Journal, $28(3), 434-440$.

\section{Appendix A. Model Identification Calculations}

Stable closed-loop transfer function:

$$
\frac{y(s)}{y_{s}(s)}=\frac{K_{2}\left(1+\tau_{z} s\right)}{\tau^{2} s^{2}+2 \zeta \tau s+1}
$$

The Laplace inverse (time-domain) of the transfer function in (A.1) is given in Yuwana and Seborg (1982) as

$$
y(t)=\Delta y_{s} K_{2}[1+D \exp (-\zeta t / \tau) \sin (E t+\phi)],
$$

where

$$
\begin{gathered}
D=\frac{\left[1-\frac{2 \zeta \tau_{z}}{\tau}+\left(\frac{\tau_{z}}{\tau}\right)^{2}\right]^{\frac{1}{2}}}{\sqrt{1-\zeta^{2}}} \\
E=\frac{\sqrt{1-\zeta^{2}}}{\tau} \\
\phi=\tan ^{-1}\left[\frac{\tau \sqrt{1-\zeta^{2}}}{\zeta \tau-\tau_{z}}\right]
\end{gathered}
$$


By differentiating (A.2) with respect to time and setting the derivative equation to zero, one gets time of the first peak:

$$
t_{p}=\frac{\tan ^{-1}\left(\frac{1-\zeta^{2}}{\zeta}\right)+\pi-\phi}{\sqrt{1-\zeta^{2}} / \tau}
$$

And the time between the first peak (overshoot) and the undershoot:

$$
t_{u}=\pi \tau / \sqrt{1-\zeta^{2}}
$$

The damping ratio $\zeta$ can be estimated as

$$
\hat{\zeta}=\frac{-\ln v}{\sqrt{\pi^{2}+(\ln v)^{2}}}
$$

where

$$
v=\frac{\Delta y_{\infty}-\Delta y_{u}}{\Delta y_{p}-\Delta y_{\infty}}
$$

Then, using equation (A.7) we get

$$
\hat{\tau}=\frac{t_{u} \sqrt{1-\hat{\zeta}^{2}}}{\pi}
$$

The steady-state gain of the closed-loop system is estimated as

$$
\hat{K}_{2}=\frac{\Delta y_{\infty}}{\Delta y_{s}} .
$$

We use time of the peak $t_{p}$ and (A.6) to get an estimate of $\phi$ :

$$
\hat{\phi}=\tan ^{-1}\left[\frac{1-\hat{\zeta}^{2}}{\hat{\zeta}}\right]-\frac{t_{p} \sqrt{1-\hat{\zeta}^{2}}}{\hat{\tau}}
$$

From (A.4), we get

$$
\hat{E}=\frac{\sqrt{1-\hat{\zeta}^{2}}}{\hat{\tau}}
$$

The overshoot is defined as

$$
D_{0}=\frac{\Delta y_{p}-\Delta y_{\infty}}{\Delta y_{\infty}}
$$

By evaluating (A.2) at time of peak $t_{p}$ we get

$$
\Delta y_{p}=\Delta y_{s} \hat{K}_{2}\left[1+\hat{D} \exp \left(-\hat{\zeta} t_{p} / \hat{\tau}\right) \sin \left(\hat{E} t_{p}+\hat{\phi}\right)\right]
$$


Combining equation (A.11), (A.14) and (A.15) gives

$$
\hat{D}=\frac{D_{0}}{\exp \left(-\hat{\zeta} t_{p} / \hat{\tau}\right) \sin \left(\hat{E} t_{p}+\hat{\phi}\right)} .
$$

We can estimate the last parameter by solving (A.3):

$$
\hat{\tau}_{z}=\hat{\xi} \hat{\tau}+\sqrt{\hat{\zeta}^{2} \hat{\tau}^{2}-\hat{\tau}^{2}\left[1-\hat{D}^{2}\left(1-\hat{\zeta}^{2}\right)\right]}
$$

Then, we back-calculate to parameters of the open-loop unstable model. The steady-state gain of the open-loop model is

$$
\hat{K}=\frac{\Delta y_{\infty}}{K_{c 0}\left|\Delta y_{s}-\Delta y_{\infty}\right|}
$$

From this, we can estimate the four model parameters in equation (A.5) are

$$
\begin{gathered}
\hat{a}_{0}=\frac{1}{\hat{\tau}^{2}\left(1+K_{c 0} \hat{K}_{p}\right)} \\
\hat{b}_{0}=\hat{K}_{p} \hat{a}_{0} \\
\hat{b}_{1}=\frac{\hat{K}_{2} \hat{\tau}_{z}}{K_{c 0} \hat{\tau}^{2}} \\
\hat{a}_{1}=-2 \hat{\zeta} / \hat{\tau}+K_{c 0} \hat{b}_{1},
\end{gathered}
$$

where $\hat{a}_{1}>0$ gives an unstable system. 